\title{
The choice of the intravenous fluid influences the tolerance of acute normovolemic anemia in anesthetized domestic pigs
}

\author{
Andreas Pape ${ }^{1 *}$, Saskia Kutschker ${ }^{1}$, Harry Kertscho', Peter Stein ${ }^{1}$, Oliver Horn', Mischa Lossen ${ }^{1}$, Bernhard Zwissler ${ }^{2}$
} and Oliver Habler ${ }^{3}$

\begin{abstract}
Introduction: The correction of hypovolemia with acellular fluids results in acute normovolemic anemia. Whether the choice of the infusion fluid has an impact on the maintenance of oxygen $\left(\mathrm{O}_{2}\right)$ supply during acute normovolemic anemia has not been investigated so far.

Methods: Thirty-six anesthetized and mechanically ventilated pigs were hemodiluted to their physiological limit of anemia tolerance, reflected by the individual critical hemoglobin concentration $\left(\mathrm{Hb}_{\text {crit }}\right)$. $\mathrm{Hb}_{\text {crit }}$ was defined as the $\mathrm{Hb}$-concentration corresponding with the onset of supply-dependency of total body $\mathrm{O}_{2}$-consumption $\left(\mathrm{VO}_{2}\right)$. The hemodilution protocol was randomly performed with either tetrastarch (6\% HES 130/0.4, TS-group, $n=9$ ), gelatin (3.5\% urea-crosslinked polygeline, GEL-group, $n=9$ ), hetastarch (6\% HES 450/0.7, HS-group, $n=9$ ) or Ringer's solution (RS-group, $n=9$ ). The primary endpoint was the dimension of $\mathrm{Hb}_{\text {crit, }}$, secondary endpoints were parameters of central hemodynamics, $\mathrm{O}_{2}$ transport and tissue oxygenation.

Results: In each animal, normovolemia was maintained throughout the protocol. $\mathrm{Hb}_{\text {crit }}$ was met at $3.7 \pm 0.6 \mathrm{~g} / \mathrm{dl}$ (RS), $3.0 \pm 0.6 \mathrm{~g} / \mathrm{dl}$ (HS $P<0.05$ vs. RS), $2.7 \pm 0.6 \mathrm{~g} / \mathrm{dl}$ (GEL, $P<0.05$ vs. RS) and $2.1 \pm 0.4 \mathrm{~g} / \mathrm{dl}(\mathrm{TS}, P<0.05$ vs. GEL, HS and RS). Hemodilution with RS resulted in a significant increase of extravascular lung water index (EVLWI) and a decrease of arterial oxygen partial pressure $\left(\mathrm{paO}_{2}\right)$, and $\mathrm{O}_{2}$ extraction ratio was increased, when animals of the TS-, GEL- and HS-groups met their individual $\mathrm{Hb}_{\text {crit. }}$.

Conclusions: The choice of the intravenous fluid has an impact on the tolerance of acute normovolemic anemia induced by acellular volume replacement. Third-generation tetrastarch preparations (e.g., HES 130/0.4) appear most advantageous regarding maintenance of tissue oxygenation during progressive anemia. The underlying mechanism includes a lower degree of extravasation and favourable effects on microcirculatory function.
\end{abstract}

\section{Introduction}

The correction of hypovolemia is an essential goal in the treatment of critically ill patients. However, the use of acellular fluids (that is, crystalloid or colloidal solutions) results in the dilution of the circulating cell mass (acute normovolemic anemia) with a corresponding decrease of $\mathrm{O}_{2}$ transport capacity [1-3].

Acute normovolemic anemia is initially compensated by increases of cardiac output and arterio-venous $\mathrm{O}_{2}$

\footnotetext{
* Correspondence: a.pape@em.uni-frankfurt.de

${ }^{1}$ Clinic of Anesthesiology, Intensive Care Medicine and Pain Management, J. W. Goethe-University Hospital Frankfurt, Theodor-Stern-Kai 7, Frankfurt/Main, 60590, Germany

Full list of author information is available at the end of the article
}

extraction [4]. Moreover, $\mathrm{O}_{2}$ supply $\left(\mathrm{DO}_{2}\right)$ physiologically exceeds $\mathrm{O}_{2}$ demand (reflected by total body $\mathrm{O}_{2}$ consumption $\left(\mathrm{VO}_{2}\right)$ under resting conditions) by the factor three to four. When $\mathrm{DO}_{2}$ begins to decrease at lower $\mathrm{Hb}$ concentrations, the total body $\mathrm{O}_{2}$ demand is still met, and $\mathrm{VO}_{2}$ remains constant despite decreasing $\mathrm{Hb}$ concentrations $\left(\mathrm{O}_{2}\right.$ supply-independency of $\left.\mathrm{VO}_{2}\right)$. When $\mathrm{DO}_{2}$ falls below a critical value, the amount of $\mathrm{O}_{2}$ delivered to the tissues becomes insufficient to meet their $\mathrm{O}_{2}$ demand and $\mathrm{VO}_{2}$ starts to decline $\left(\mathrm{O}_{2}\right.$ supplydependency of $\mathrm{VO}_{2}$ ) [5]. The corresponding critical hemoglobin concentration $\left(\mathrm{Hb}_{\text {crit }}\right)$ represents the ultimate limit of the individual anemia tolerance.

\section{Ciomed Central}

(c) 2012 Pape et al.; licensee BioMed Central Ltd. This is an open access article distributed under the terms of the Creative Commons Attribution License (http://creativecommons.org/licenses/by/2.0), which permits unrestricted use, distribution, and reproduction in any medium, provided the original work is properly cited. 
Among clinicians all over the world, infusion practice varies considerably and different intravenous (IV) fluids are used for volume replacement [6]. However, currently available IV fluids differ in their pharmacodynamic and kinetic profile (for example, molecular weight, plasma half-life, volume expansion effect) and in their effects on rheology and microcirculatory function.

We hypothesized that these properties might have an influence on the tolerance of acute normovolemic anemia. In this regard, the present study was conceived to compare potential effects of four commonly used IV fluids: low-molecular hydroxyethyl starch (HES) (tetrastarch, 6\% HES 130/0.4), high-molecular HES (hetastarch, 6\% HES 450/0.7), gelatin (3.5\% urea-crosslinked polygeline), and crystalloid volume replacement with Ringer's solution.

\section{Materials and methods}

After approval by the local governmental review board (Regional Council Darmstadt, department for veterinary affairs/V54), experiments were performed in 36 healthy farm-bred pigs of either sex (body weight $24.0 \pm 3.7 \mathrm{~kg}$ ). All animals received good care in compliance with the Guide for the Care and Use of Laboratory Animals.

\section{Anesthesia and ventilation}

Animals were denied food 12 hours before each experiment started, but had free access to water. After intramuscular premedication with $10 \mathrm{mg} / \mathrm{kg}$ ketamine (Ketavet $^{\mathrm{TM}}$, Parke-Davis, Berlin, Germany) and $1 \mathrm{mg} / \mathrm{kg}$ midazolam (Midazolam ${ }^{\mathrm{TM}}$, Ratiopharm, Ulm, Germany), anesthesia was induced by IV injection of $3 \mathrm{mg} / \mathrm{kg}$ propofol (Propofol ${ }^{\mathrm{TM}}$, Braun, Melsungen, Germany) and 30 $\mu \mathrm{g} / \mathrm{kg}$ fentanyl (Fentanyl ${ }^{\mathrm{TM}}$, Janssen, Neuss, Germany) and maintained by continuous infusion of propofol $(0.16$ $\mathrm{mg} / \mathrm{kg} / \mathrm{min})$, midazolam $(0.01 \mathrm{mg} / \mathrm{kg} / \mathrm{min})$ and fentanyl $(0.8 \mu \mathrm{g} / \mathrm{kg} / \mathrm{min})$. To facilitate ventilation, muscular paralysis was achieved with pancuronium bromide (Pancuronium $^{\mathrm{TM}}$, DeltaSelect, Dreieich, Germany, bolus injection $0.2 \mathrm{mg} / \mathrm{kg}$ after induction of anesthesia, followed by continuous infusion of $0.13 \mathrm{mg} / \mathrm{kg} / \mathrm{min}$ ). Estimated fluid losses were replaced with Ringer's solution (Ringer-Infusionslösung ${ }^{\mathrm{TM}}$, Braun, Melsungen, Germany, $3 \mathrm{~mL} / \mathrm{kg} / \mathrm{h}$ ).

Animals were orotracheally intubated and ventilated with ambient air at a rate of 14 cycles/min and a positive end-expiratory pressure of $5 \mathrm{cmH}_{2} \mathrm{O}$ (Servo 900B, SiemensElema, Solna, Sweden). Tidal volume was individually adjusted to provide arterial normocapnia and was then maintained throughout the entire protocol.

\section{Instrumentation and monitoring}

Animals were placed in the supine position and a fivelead electrocardiogram (II, V5) was installed for detection of arrhythmias and ST-segment changes. A double-lumen catheter (Arrow, Reading, PA, USA) was inserted into the cranial vena cava and a Swan-GanzCatheter (Baxter, Irvine, CA, USA) was floated into a branch of the pulmonary artery. Each one $6 \mathrm{~F}$ introducer sheath (Arrow, Reading, PA, USA) was inserted into the right femoral vein and artery, respectively. For continuous measurement of arterial blood pressure and cardiac output, a thermodilution catheter was placed into the left femoral artery (Pulsion Medical Systems, Munich, Germany). Body core temperature was kept constant using a warming pad.

\section{Experimental protocol}

Upon completion of catheter insertion and installation of the different measuring devices, a $60 \mathrm{~min}$ stabilization period was allowed to elapse before the first data set was recorded (baseline). Subsequently, animals were randomized to undergo acute normovolemic hemodilution with one of the following fluids: 1) 6\% HES 130/0.4 (tetrastarch, TS-group, $n=9$ ); 2) 6\% HES 450/0.7 (hetastarch, HS-group, $n=9$ ); 3) 3.5\% urea-crosslinked polygeline (gelatin, GEL-group, $n=9$ ), or 4) Ringer's solution (RS-group, $n=9$ ).

Acute normovolemic anemia was induced by withdrawal of blood $(1 \mathrm{~mL} / \mathrm{kg} / \mathrm{min})$ and simultaneous infusion of one of the IV fluids. To maintain normovolemia during the hemodilution protocol, the following infusion rates were chosen with regard to the different plasma expansion effects of the fluids investigated (see Table 1): Ringer's solution $3 \mathrm{~mL} / \mathrm{kg} / \mathrm{min}$, gelatin $1.2 \mathrm{~mL} / \mathrm{kg} / \mathrm{min}$, tetrastarch and hetastarch $1 \mathrm{~mL} / \mathrm{kg} / \mathrm{min}$, respectively. Infusion fluids were warmed in an immersion bath until IV infusion. For precise synchronization of blood withdrawal with the corresponding infusion rates, a bidirectional precision pump (Harvard Apparatus, Holliston, MA, USA) was used.

The target parameter of the hemodilution protocol was the animal's individual critical $\mathrm{Hb}$ concentration $\left(H b_{\text {crit }}\right)$, which was defined as the $\mathrm{Hb}$ concentration corresponding with a critical limitation of $\mathrm{O}_{2}$ delivery to the tissues $\left(\mathrm{DO}_{2}\right)$ and the onset of $\mathrm{O}_{2}$ supply-dependency of total body $\mathrm{O}_{2}$ consumption- $\mathrm{VO}_{2}$ (see below). At the end of the hemodilution protocol, animals were killed by intracardiac injection of saturated potassium chloride solution.

\section{Measurements}

Intravascular blood volume was determined at baseline using the "whole-blood" method of the indocyanin green (ICG) indicator dilution technique, which has already been described in detail elsewhere [7]. The pressure transducers of the cardiovascular catheters were connected with a multi-channel recorder and a personal 
Table 1 Characteristics and trade names of intravenous fluids investigated in the present study [25].

\begin{tabular}{|c|c|c|c|c|}
\hline & Tetrastarch & Hetastarch & Gelatin & Ringer's solution \\
\hline Concentration (\%) & 6 & 6 & 3.5 & n.a. \\
\hline $\begin{array}{l}\text { Mean molecular } \\
\text { weight }(\mathrm{kDa})\end{array}$ & 130 & 450 & 35 & n.a. \\
\hline Volume efficacy (\%) & 100 & 100 & 80 & 30 \\
\hline Volume effect (min) & $120-180$ & $300-360$ & $60-180$ & 20 \\
\hline Viscosity (cp) & 2.1 & 3.9 & 1.7 & n.a. \\
\hline $\mathrm{COP}$ in vitro $(\mathrm{mmHg})$ & 36 & 26 & 25 & n.a. \\
\hline Trade name & $\begin{array}{c}\text { Voluven }{ }^{\mathrm{TM}} \text {, Fresenius Kabi, Bad } \\
\text { Homburg, Germany }\end{array}$ & $\begin{array}{l}\text { HESPANTM, } \\
\text { Braun, } \\
\text { Irvine, CA, } \\
\text { USA }\end{array}$ & $\begin{array}{c}\text { Haemacce|TM, DeltaSelect, } \\
\text { Dreieich, } \\
\text { Germany }\end{array}$ & $\begin{array}{c}\text { Ringer-Infusions-lösung }{ }^{\mathrm{TM}} \text {, Braun } \\
\text { Melsungen, Germany }\end{array}$ \\
\hline
\end{tabular}

COP, colloidal oncotic pressure; n.a., not applicable.

computer (Hugo-Sachs, March-Hugstetten, Germany) for continuous measurement of hemodynamic parameters. Cardiac output was continuously measured using the pulse contour analysis (PICCO classic monitor, Pulsion Medical Systems, Munich, Germany). The volumetric preload and extravasation parameters, intrathoracic blood volume (ITBV) and extravascular lung water (EVLW), were assessed after exchange of each $10 \%$ of the circulating blood volume using the transpulmonary thermodilution technique. Arterial and mixed venous blood samples were withdrawn at baseline, after exchange of each $10 \%$ of the circulating blood volume and at $\mathrm{Hb}_{\text {crit }}$ for blood gas analysis and assessment of $\mathrm{Hb}$ concentration (GEM-3000 and $682 \mathrm{CO}-\mathrm{Oxymeter}$, Instrumentation Laboratory, Lexington, MA, USA).

\section{Calculated parameters}

Body surface area (BSA) was calculated according to Holt [8], from body weight (BW) and a species-dependent constant:

$$
B S A=k \cdot B W^{\frac{2}{3}}\left(m^{2}\right)
$$

where $\mathrm{k}=9$ for the species pig.

The measured values of cardiac output (CO) and $\mathrm{O}_{2}$ consumption $\left(\mathrm{VO}_{2}\right)$ were indexed to BSA:

$$
\begin{aligned}
& C I=\frac{C O}{B S A} \quad\left(\frac{l}{\min \cdot m^{2}}\right) \\
& V O_{2} I=\frac{V O_{2}}{B S A} \quad\left(\frac{m l}{\min \cdot m^{2}}\right)
\end{aligned}
$$

Systemic and pulmonary vascular resistance indices (SVRI and PVRI) were calculated from standard formulae:

$$
S V R I=\frac{(M A P-C V P) \cdot 79.9}{C I} \quad\left(\frac{d y n \cdot s}{c m^{5} \cdot m^{2}}\right)
$$

$$
P V R I=\frac{(M P A P-P C W P) \cdot 79.9}{C I} \quad\left(\frac{d y n \cdot s}{\mathrm{~cm}^{5} \cdot \mathrm{m}^{2}}\right)
$$

where MAP = mean arterial pressure, $\mathrm{CVP}=$ central venous pressure, $\mathrm{CI}=$ cardiac index, $\mathrm{MPAP}=$ mean pulmonary arterial pressure and PCWP = pulmonary capillary wedge pressure.

Arterial and mixed venous $\mathrm{O}_{2}$ content $\left(\mathrm{CaO}_{2}\right.$ and $\left.\mathrm{CvO}_{2}\right), \mathrm{O}_{2}$ delivery $\left(\mathrm{DO}_{2} \mathrm{I}\right)$ and $\mathrm{O}_{2}$ extraction ratio were calculated as follows:

$$
\begin{aligned}
& \mathrm{CaO}_{2}=[\mathrm{Hb}] \cdot 1.34 \cdot \mathrm{SaO}_{2}+0.0031 \cdot \mathrm{paO}_{2} \quad\left(\frac{m L}{d L}\right) \\
& \mathrm{CvO}_{2}=[\mathrm{Hb}] \cdot 1.34 \cdot \mathrm{SvO} \mathrm{O}_{2}+0.0031 \cdot p v \mathrm{O}_{2} \quad\left(\frac{m L}{d L}\right) \\
& \mathrm{DO}_{2} \mathrm{I}=\mathrm{CI} \cdot \mathrm{CaO} 2\left(\frac{m L}{\mathrm{~min} \cdot m^{2}}\right) \\
& \mathrm{O}_{2}-\mathrm{ER}=\frac{\mathrm{CaO}_{2}-\mathrm{CvO}_{2}}{\mathrm{CaO}_{2}}(\%)
\end{aligned}
$$

\section{Determination of $\mathrm{Hb}_{\text {crit }}$}

$\mathrm{Hb}_{\text {crit }}$ is the correlate of the critical limitation of $\mathrm{DO}_{2}$, and marks the onset of total body $\mathrm{O}_{2}$ supply-dependency. The corresponding decrease of $\mathrm{VO}_{2}$ was prospectively detected in an automated and investigator-independent manner: $\mathrm{VO}_{2}$ was measured every $60 \mathrm{sec}$ with a metabolic monitor (Delta-Trac II'M MBM-200, Datex-Engstrom, Helsinki, Finland). $\mathrm{VO}_{2}$ values were simultaneously recorded and computed with specific software (Delta-Crit-System, DCS) [9]. During the stabilization period, the DCS enters $\mathrm{VO}_{2}$ values into an online regression analysis and calculates the mean and standard deviation (SD). During the subsequent hemodilution period, the $\mathrm{VO}_{2}$ value obtained every 60 secis compared to the mean value predicted by the DCS. 
When three consecutive $\mathrm{VO}_{2}$ values are outside the predefined range (3-fold SD of the regression line), a significant decrease of $\mathrm{VO}_{2}$ is assumed (see Figure 1) and the computer alerts visually and acoustically [9]. For determination of $\mathrm{Hb}_{\text {crit, }}$, the corresponding $\mathrm{Hb}$ concentration was measured in the arterial blood sample.

\section{Statistics}

Statistical analysis was performed with the SAS 9.1 software package (SAS Institute, Cary, NC, USA). All data are presented as mean \pm SD. Distribution of data was assessed with the Shapiro-Wilk test. In the case of normal distribution, the time effect on the different variables, and differences between groups at different time points were tested by repeated analysis of variance (ANOVA). Post hoc analysis of differences detected with ANOVA was performed with the Student Newman Keuls test. In the case of non-normal distribution, the time effect on the parameters, and the between-group differences were tested by analysis of variance on ranks (rANOVA). Post hoc analysis of differences detected with rANOVA was performed with Tukey's test. For all parameters, statistical significance was accepted at $P<0.05$.

\section{Results}

\section{Baseline characteristics}

On analysis of baseline body weight, blood volume index and all investigated parameters of hemodynamics and $\mathrm{O}_{2}$ transport, there were no significant differences detected between the groups (see Tables 2 and 3).

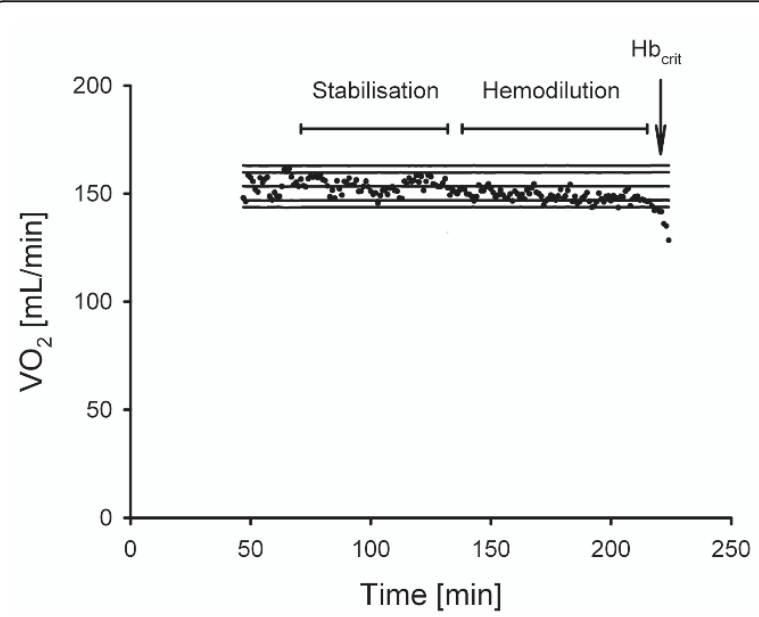

Figure 1 Typical example of a recording of total body oxygen consumption $\left(\mathrm{VO}_{2}\right)$ in the course of the experimental protocol. A linear regression analysis including the calculation of SD was performed with $\mathrm{VO}_{2}$ values collected during the 60-minute stabilization period. During the subsequent hemodilution protocol, a critical limitation of oxygen delivery $\left(\mathrm{DO}_{2}\right)$ was assumed, when three consecutive $\mathrm{VO}_{2}$ values fell below the lower $3 \sigma$-range.
Primary endpoint: critical $\mathrm{Hb}$ concentation $\left(\mathrm{Hb}_{\text {crit }}\right)$ Depending on the plasma substitute used for hemodilution, $\mathrm{Hb}_{\text {crit }}$ was met at $2.1 \pm 0.4 \mathrm{~g} / \mathrm{dL}$ (TS-group), $2.7 \pm$ $0.6 \mathrm{~g} / \mathrm{dL}$ (GEL-group), $3.0 \pm 0.6 \mathrm{~g} / \mathrm{dL}$ (HS-group) and $3.7 \pm 0.6 \mathrm{~g} / \mathrm{dL}$ (RS-group). The lowest value of $\mathrm{Hb}_{\text {crit }}$ was attained in the TS-group $(P<0.05$ vs. GEL, HS and $\mathrm{RS})$. While $\mathrm{Hb}_{\text {crit }}$ did not differ significantly between the GEL- and HS-groups, the difference between the RSgroup and the other groups (GEL-, HS- and TS- groups) was statistically significant (see Figure 2).

The induction of critical normovolemic anemia required the exchange of $69 \pm 21 \%$ of the circulating blood volume (BV) for Ringer's solution, $71 \pm 19 \%$ BV for hetastarch, $93 \pm 44 \% \mathrm{BV}$ for gelatin $(P<0.05$ vs. HS- and RS-groups) and $107 \pm 28 \% \mathrm{BV}$ for tetrastarch $(P<0.05$ vs. HS- and RS-groups). For maintenance of normovolemia, animals received 1,812 $\pm 535 \mathrm{~mL}$ tetrastarch, 2,016 $\pm 898 \mathrm{~mL}$ gelatin, $1,369 \pm 324 \mathrm{~mL}$ hetastarch $(P<0.05$ vs. TS-group $)$, or $3,698 \pm 1320 \mathrm{~mL}$ Ringer's solution, respectively $(P<0.05$ vs. TS- GELand HS-groups).

\section{Secondary endpoints: hemodynamic and $\mathrm{O}_{2}$-derived parameters}

In Tables 2 and 3, hemodynamic and $\mathrm{O}_{2}$-derived parameters are presented at the respective critical $\mathrm{Hb}$ concentrations, that is, after hemodilution to $3.7 \mathrm{~g} / \mathrm{dL}, 3.0$ $\mathrm{g} / \mathrm{dL}, 2.7 \mathrm{~g} / \mathrm{dL}$ and $2.1 \mathrm{~g} / \mathrm{dL}$, respectively.

Hemodilution to $\mathrm{Hb} 3.7 \mathrm{~g} / \mathrm{dL}$ ( $H b_{\text {crit }}$ of the $\mathrm{RS}$-group)

As compensation for hemodilution, cardiac index (CI) increased by 46 to $60 \%$ in all groups $(P<0.05$ vs. baseline). Consistently, stroke volume index (SVI) and heart rate (HR) increased by 24 to $40 \%$ and 14 to $18 \%$, respectively. While the increases in SVI were significant in the TS-group, GEL-, and RS-groups, the increase in HR was not significant in any group. Mean arterial pressure (MAP) decreased by 11 to $21 \%$ in the GEL-, HS- and RS-groups (non-significant) and systemic vascular resistance index (SVRI) decreased in all groups by 35 to $46 \%$ $(P<0.05$ vs. baseline $)$, while mean pulmonary arterial pressure (MPAP) increased by 22 to $50 \%(P<0.05$ vs. baseline in the GEL-, HS and RS-group). Stroke volume variation (SVV) remained unchanged in the RS-group and tended to decrease in the TS- and GEL-groups. In the HS-group, the decrease in SVV was statistically significant. Compared with baseline, ITBV index (ITBVI) increased in all groups $(P<0.05)$, reflecting increased venous return to the heart. The ITBVI was higher in animals hemodiluted with TS or HS than after fluid replacement with $\mathrm{RS}(P<0.05)$. As a consequence of hemodilution, $\mathrm{DO}_{2} \mathrm{I}$ decreased by 14 to $30 \%$ in all groups $(P<0.05$ vs. baseline $)$. Moreover, the $\mathrm{pH}$-value and base excess (BE) decreased significantly in the GEL, HS- and RS-groups. As the protocol was terminated at 
Table 2 Hemodynamic parameters obtained at baseline and after hemodilution to the individual $\mathrm{Hb}_{\text {crit }}$ of the RL-, HS-, GEL- and TS-groups.

\begin{tabular}{|c|c|c|c|c|c|c|c|c|c|c|}
\hline \multirow{3}{*}{$\begin{array}{c}\text { Parameter } \\
\text { BVI } \\
(\mathrm{m} / / \mathrm{kg})\end{array}$} & \multirow{2}{*}{$\frac{\text { Group }}{\mathrm{TS}}$} & \multirow{2}{*}{$\begin{array}{c}\text { Baseline } \\
73 \pm 6\end{array}$} & \multicolumn{2}{|c|}{$\mathrm{Hb}_{\text {crit }} \mathrm{RS}$-group } & \multicolumn{2}{|c|}{$\mathrm{Hb}_{\text {crit }} \mathrm{HS}$-group } & \multicolumn{2}{|c|}{$\mathrm{Hb}_{\text {crit }}$ GEL-group } & \multicolumn{2}{|c|}{$\mathrm{Hb}_{\text {crit }} \mathrm{TS}$-group } \\
\hline & & & n.a. & & n.a. & & n.a. & & n.a. & \\
\hline & GEL & $76 \pm 9$ & n.a. & & n.a. & & n.a. & & n.a. & \\
\hline & $\mathrm{HS}$ & $73 \pm 3$ & n.a. & & n.a. & & n.a. & & n.a. & \\
\hline & RS & $76 \pm 8$ & n.a. & & n.a. & & n.a. & & n.a. & \\
\hline $\mathrm{HR}$ & TS & $102 \pm 15$ & $115 \pm 19$ & & $120 \pm 16$ & & $132 \pm 25$ & + & $133 \pm 29$ & + \\
\hline \multirow[t]{3}{*}{$(1 / \mathrm{min})$} & GEL & $100 \pm 23$ & $111 \pm 16$ & & $114 \pm 15$ & & $126 \pm 15$ & + & n.a. & \\
\hline & $\mathrm{HS}$ & $102 \pm 19$ & $120 \pm 17$ & & $121 \pm 8$ & + & n.a. & & n.a. & \\
\hline & RS & $111 \pm 20$ & $127 \pm 35$ & & n.a. & & n.a. & & n.a. & \\
\hline MAP & TS & $81 \pm 14$ & $88 \pm 18$ & & $84 \pm 18$ & & $80 \pm 19$ & & $59 \pm 15$ & \\
\hline \multirow[t]{3}{*}{$(\mathrm{mmHg})$} & GEL & $88 \pm 12$ & $78 \pm 14$ & & $77 \pm 15$ & & $64 \pm 12$ & + & n.a. & \\
\hline & HS & $88 \pm 20$ & $75 \pm 13$ & & $70 \pm 13$ & + & n.a. & & n.a. & \\
\hline & RS & $90 \pm 20$ & $71 \pm 10$ & & n.a. & & n.a. & & n.a. & \\
\hline MPAP & TS & $19 \pm 4$ & $23 \pm 4$ & & $23 \pm 4$ & & $23 \pm 4$ & & $23 \pm 5$ & \\
\hline \multirow[t]{3}{*}{$(\mathrm{mmHg})$} & GEL & $19 \pm 3$ & $25 \pm 6$ & + & $24 \pm 5$ & + & $26 \pm 5$ & + & n.a. & \\
\hline & $\mathrm{HS}$ & $18 \pm 3$ & $22 \pm 6$ & + & $24 \pm 4$ & + & n.a. & & n.a. & \\
\hline & RS & $18 \pm 3$ & $27 \pm 11$ & + & n.a. & & n.a. & & n.a. & \\
\hline $\mathrm{Cl}$ & TS & $4.2 \pm 0.5$ & $6.7 \pm 0.8$ & + & $7.5 \pm 1.2$ & + & $7.7 \pm 1.1$ & + & $8.0 \pm 2.8$ & + \\
\hline \multirow[t]{3}{*}{$\left(\mathrm{L} / \mathrm{min} / \mathrm{m}^{2}\right)$} & GEL & $4.6 \pm 0.8$ & $6.7 \pm 0.7$ & + & $7.5 \pm 0.5$ & + & $7.5 \pm 0.9$ & + & n.a. & \\
\hline & HS & $4.5 \pm 0.5$ & $6.7 \pm 0.6$ & + & $7.0 \pm 1.1$ & + & n.a. & & n.a. & \\
\hline & RS & $4.4 \pm 0.8$ & $6.7 \pm 0.8$ & + & n.a. & & n.a. & & n.a. & \\
\hline SVI & TS & $42 \pm 8$ & $59 \pm 11$ & + & $63 \pm 11$ & + & $59 \pm 11$ & + & $57 \pm 3$ & + \\
\hline \multirow[t]{3}{*}{$\left(\mathrm{mL} / \mathrm{m}^{2}\right)$} & GEL & $47 \pm 7$ & $62 \pm 12$ & + & $67 \pm 10$ & + & $61 \pm 9$ & & n.a & \\
\hline & $\mathrm{HS}$ & $45 \pm 9$ & $56 \pm 7$ & & $55 \pm 11$ & & n.a & & n.a & \\
\hline & RS & $41 \pm 9$ & $55 \pm 13$ & + & n.a. & & n.a. & & n.a. & \\
\hline CVP & TS & $6 \pm 4$ & $7 \pm 0$ & & $8 \pm 3$ & & $8 \pm 2$ & & $8 \pm 1$ & \\
\hline \multirow[t]{3}{*}{$(\mathrm{mmHg})]$} & GEL & $5 \pm 3$ & $8 \pm 3$ & & $8 \pm 3$ & & $8 \pm 3$ & & n.a. & \\
\hline & $\mathrm{HS}$ & $6 \pm 3$ & $9 \pm 4$ & & $10 \pm 6$ & & n.a. & & n.a. & \\
\hline & RS & $4 \pm 2$ & $7 \pm 3$ & + & n.a. & & n.a. & & n.a. & \\
\hline PCWP & TS & $5 \pm 3$ & $7 \pm 2$ & & $6 \pm 2$ & & $7 \pm 2$ & & $8 \pm 2$ & \\
\hline \multirow[t]{3}{*}{$(\mathrm{mmHg})$} & GEL & $6 \pm 2$ & $6 \pm 3$ & & $7 \pm 3$ & & $7 \pm 3$ & & n.a. & \\
\hline & $\mathrm{HS}$ & $4 \pm 3$ & $5 \pm 2$ & & $5 \pm 3$ & & n.a. & & n.a. & \\
\hline & RS & $4 \pm 3$ & $6 \pm 3$ & & n.a. & & n.a. & & n.a. & \\
\hline ITBVI & TS & $673 \pm 65$ & $857 \pm 142$ & $+\S$ & $856 \pm 63$ & + & $844 \pm 78$ & $+^{*}$ & $764 \pm 177$ & \\
\hline \multirow[t]{3}{*}{$\left(\mathrm{mL} / \mathrm{m}^{2}\right)$} & GEL & $699 \pm 94$ & $818 \pm 101$ & + & $824 \pm 82$ & + & $784 \pm 57$ & + & n.a. & \\
\hline & HS & $664 \pm 78$ & $829 \pm 84$ & $+\S$ & $774 \pm 86$ & + & n.a. & & n.a. & \\
\hline & RS & $699 \pm 34$ & $761 \pm 70$ & + & n.a. & & n.a. & & n.a. & \\
\hline SW & TS & $21 \pm 6$ & $13 \pm 3$ & & $10 \pm 3$ & + & $11 \pm 3$ & $+^{*}$ & $10 \pm 2$ & + \\
\hline \multirow[t]{3}{*}{ (\%) } & GEL & $19 \pm 5$ & $12 \pm 3$ & & $10 \pm 3$ & + & $16 \pm 4$ & & n.a. & \\
\hline & $\mathrm{HS}$ & $20 \pm 6$ & $14 \pm 2$ & $+\S$ & $13 \pm 3$ & + & n.a. & & n.a. & \\
\hline & RS & $17 \pm 1$ & $18 \pm 7$ & & n.a. & & n.a. & & n.a. & \\
\hline & TS & $1447 \pm 342$ & $943 \pm 178$ & + & $817 \pm 212$ & + & $751 \pm 204$ & + & $517 \pm 126$ & + \\
\hline \multirow[t]{3}{*}{$\left(\right.$ dyn $\left./ \mathrm{sec} / \mathrm{cm}^{5} / \mathrm{m}^{2}\right)$} & GEL & $1489 \pm 433$ & $834 \pm 180$ & + & $736 \pm 181$ & + & $603 \pm 147$ & + & n.a. & \\
\hline & $\mathrm{HS}$ & $1490 \pm 386$ & $801 \pm 236$ & + & $590 \pm 135$ & + & n.a. & & n.a. & \\
\hline & RS & $1578 \pm 439$ & $871 \pm 221$ & + & n.a. & & n.a. & & n.a. & \\
\hline
\end{tabular}

$\mathrm{BVI}$, blood volume indexed to body weight (only assessed at baseline); $\mathrm{HR}$, heart rate; $\mathrm{MAP}$, mean arterial pressure; $\mathrm{MPAP}$, mean pulmonary arterial pressure; $\mathrm{Cl}$ cardiac index; SVI, stroke volume index; CVP, central venous pressure; PCWP, pulmonary capillary wedge pressure; ITBVI, intrathoracic blood volume index; SVV, stroke volume variation; SVRI, systemic vascular resistance index; n.a., not applicable. ${ }^{*} P<0.05$ vs. gelatin, \#P<0.05 vs. HS, $\S P<0.05$ vs. RS, $+P<0.05$ vs. baseline. 
Table 3 Oxygen-derived parameters investigated at baseline and after hemodilution to the individual $\mathrm{Hb}_{\text {crit }}$ of the RL-, HS-, GEL- and TS-groups.

\begin{tabular}{|c|c|c|c|c|c|c|c|c|c|c|}
\hline \multirow{2}{*}{$\begin{array}{c}\text { Parameter } \\
\mathrm{Hb}\end{array}$} & \multirow{2}{*}{$\frac{\text { Group }}{\mathrm{TS}}$} & \multirow{2}{*}{$\begin{array}{l}\text { Baseline } \\
7.6 \pm 0.6\end{array}$} & \multicolumn{2}{|c|}{$\mathrm{Hb}_{\text {crit }} \mathrm{RS}$-group } & \multicolumn{2}{|c|}{$\mathrm{Hb}_{\text {crit }} \mathrm{HS}$-group } & \multicolumn{2}{|c|}{$\mathrm{Hb}_{\text {crit }}$ GEL-group } & \multicolumn{2}{|c|}{$\mathrm{Hb}_{\text {crit }}$ TS-group } \\
\hline & & & $3.7 \pm 0.2$ & + & $3.0 \pm 0.1$ & + & $2.7 \pm 0.1$ & + & $2.1 \pm 0.4$ & + \\
\hline \multirow[t]{3}{*}{ (g/dL) } & GEL & $7.7 \pm 0.5$ & $3.6 \pm 0.3$ & + & $2.9 \pm 0.2$ & + & $2.7 \pm 0.7$ & + & n.a. & \\
\hline & $\mathrm{HS}$ & $7.6 \pm 0.4$ & $3.7 \pm 0.3$ & + & $3.0 \pm 0.6$ & + & n.a. & & n.a. & \\
\hline & RS & $7.9 \pm 0.5$ & $3.7 \pm 0.6$ & + & n.a. & & n.a. & & n.a. & \\
\hline Temp. & TS & $37.8 \pm 1.3$ & $37.5 \pm 0.5$ & & $37.6 \pm 0.4$ & & $37.7 \pm 1.2$ & & $37.6 \pm 1.2$ & \\
\hline \multirow[t]{3}{*}{$\left({ }^{\circ} \mathrm{C}\right)$} & GEL & $37.5 \pm 1.0$ & $37.4 \pm 1.2$ & & $37.3 \pm 0.8$ & & $37.2 \pm 1.0$ & & n.a. & \\
\hline & $\mathrm{HS}$ & $37.6 \pm 1.1$ & $37.6 \pm 1.0$ & & $37.4 \pm 1.2$ & & n.a. & & n.a. & \\
\hline & RS & $37.7 \pm 0.7$ & $37.4 \pm 0.6$ & & n.a. & & n.a. & & n.a. & \\
\hline $\mathrm{paO}_{2}$ & TS & $94 \pm 9$ & $95 \pm 12$ & $\S$ & $100 \pm 13$ & & $98 \pm 14$ & & $105 \pm 22$ & \\
\hline \multirow[t]{3}{*}{$(\mathrm{mmHg})$} & GEL & $98 \pm 10$ & $99 \pm 19$ & $\S$ & $101 \pm 14$ & & $95 \pm 18$ & & n.a. & \\
\hline & $\mathrm{HS}$ & $95 \pm 7$ & $98 \pm 9$ & $\S$ & $101 \pm 11$ & & n.a. & & n.a. & \\
\hline & RS & $100 \pm 8$ & $79 \pm 10$ & + & n.a. & & n.a. & & n.a. & \\
\hline $\mathrm{paCO}_{2}$ & TS & $34 \pm 3$ & $35 \pm 4$ & & $34 \pm 4$ & & $34 \pm 4$ & & $33 \pm 4$ & \\
\hline \multirow[t]{3}{*}{$(\mathrm{mmHg})$} & GEL & $34 \pm 2$ & $35 \pm 3$ & & $34 \pm 4$ & & $35 \pm 4$ & & n.a. & \\
\hline & $\mathrm{HS}$ & $33 \pm 4$ & $33 \pm 3$ & & $33 \pm 3$ & & n.a. & & n.a. & \\
\hline & RS & $32 \pm 3$ & $32 \pm 4$ & & n.a. & & n.a. & & n.a. & \\
\hline $\mathrm{pvO}_{2}$ & TS & $34 \pm 3$ & $31 \pm 5$ & & $32 \pm 4$ & & $31 \pm 4$ & & $29 \pm 4$ & + \\
\hline \multirow[t]{3}{*}{$(\mathrm{mmHg})$} & GEL & $34 \pm 2$ & $32 \pm 3$ & & $34 \pm 2$ & \# & $32 \pm 4$ & & n.a. & \\
\hline & $\mathrm{HS}$ & $33 \pm 4$ & $35 \pm 4$ & & $29 \pm 3$ & + & n.a. & & n.a. & \\
\hline & RS & $32 \pm 3$ & $34 \pm 3$ & & n.a. & & n.a. & & n.a. & \\
\hline $\mathrm{DO}_{2} \mathrm{I}$ & TS & $428 \pm 45$ & $367 \pm 58$ & + & $324 \pm 54$ & + & $287 \pm 20$ & + & $253 \pm 84$ & + \\
\hline \multirow[t]{3}{*}{$\left(\mathrm{mL} / \mathrm{min} / \mathrm{m}^{2}\right)$} & GEL & $478 \pm 67$ & $348 \pm 51$ & + & $328 \pm 35$ & + & $289 \pm 44$ & + & n.a. & \\
\hline & $\mathrm{HS}$ & $455 \pm 53$ & $353 \pm 22$ & + & $303 \pm 65$ & + & n.a. & & n.a. & \\
\hline & RS & $487 \pm 109$ & $339 \pm 69$ & + & n.a. & & n.a. & & n.a. & \\
\hline $\mathrm{VO}_{2} \mathrm{I}$ & TS & $223 \pm 33$ & $219 \pm 29$ & & $203 \pm 14$ & & $216 \pm 34$ & & $199 \pm 35$ & \\
\hline \multirow[t]{3}{*}{$\left(\mathrm{mL} / \mathrm{min} / \mathrm{m}^{2}\right)$} & GEL & $219 \pm 17$ & $210 \pm 10$ & & $201 \pm 12$ & & $199 \pm 12$ & & n.a. & \\
\hline & $\mathrm{HS}$ & $229 \pm 37$ & $199 \pm 10$ & & $211 \pm 35$ & & n.a. & & n.a. & \\
\hline & RS & $222 \pm 23$ & $203 \pm 26$ & & n.a. & & n.a. & & n.a. & \\
\hline $\mathrm{O}_{2}-\mathrm{ER}$ & TS & $37 \pm 5$ & $46 \pm 11$ & & $45 \pm 7$ & \# & $46 \pm 11$ & & $52 \pm 13$ & + \\
\hline \multirow[t]{3}{*}{ (\%) } & GEL & $35 \pm 5$ & $46 \pm 8$ & & $41 \pm 7$ & \# & $45 \pm 12$ & + & n.a. & \\
\hline & $\mathrm{HS}$ & $38 \pm 9$ & $44 \pm 6$ & & $52 \pm 9$ & + & n.a. & & n.a. & \\
\hline & RS & $39 \pm 7$ & $44 \pm 6$ & & n.a. & & n.a. & & n.a. & \\
\hline \multirow[t]{4}{*}{$\mathrm{pH}$} & TS & $7.57 \pm 0.04$ & $7.53 \pm 0.04$ & & $7.52 \pm 0.04$ & & $7.51 \pm 0.04$ & & $7.50 \pm 0.06$ & \\
\hline & GEL & $7.58 \pm 0.02$ & $7.50 \pm 0.03$ & + & $7.49 \pm 0.03$ & & $7.47 \pm 0.04$ & & n.a. & \\
\hline & $\mathrm{HS}$ & $7.59 \pm 0.04$ & $7.55 \pm 0.05$ & + & $7.53 \pm 0.04$ & & n.a. & & n.a. & \\
\hline & RS & $7.58 \pm 0.05$ & $7.42 \pm 0.08$ & + & n.a. & & n.a. & & n.a. & \\
\hline $\mathrm{BE}$ & TS & $8.7 \pm 3.4$ & $6.1 \pm 3.5$ & $\S$ & $5.0 \pm 3.4$ & & $4.2 \pm 3.4$ & + & $2.4 \pm 4.0$ & + \\
\hline \multirow[t]{3}{*}{ (mmol/L) } & GEL & $9.4 \pm 2.0$ & $4.0 \pm 1.7$ & $+\S$ & $2.7 \pm 2.1$ & + & $1.8 \pm 2.8$ & + & n.a. & \\
\hline & $\mathrm{HS}$ & $9.3 \pm 2.4$ & $6.2 \pm 3.0$ & $+\S$ & $4.6 \pm 2.5$ & + & n.a. & & n.a. & \\
\hline & RS & $7.5 \pm 2.1$ & $-3.5 \pm 3.4$ & + & n.a. & & n.a. & & n.a. & \\
\hline Lactate & TS & $1.9 \pm 0.9$ & $1.7 \pm 0.8$ & & $1.7 \pm 0.8$ & & $1.7 \pm 0.9$ & & $2.3 \pm 1.0$ & \\
\hline \multirow[t]{3}{*}{$(\mathrm{mmol} / \mathrm{L})$} & GEL & $1.4 \pm 0.3$ & $1.2 \pm 0.3$ & & $1.1 \pm 0.3$ & & $1.1 \pm 0.3$ & & n.a. & \\
\hline & $\mathrm{HS}$ & $1.7 \pm 0.7$ & $1.6 \pm 0.5$ & & $1.8 \pm 0.1$ & & n.a. & & n.a. & \\
\hline & RS & $1.4 \pm 0.6$ & $1.3 \pm 0.6$ & & n.a. & & n.a. & & n.a. & \\
\hline
\end{tabular}

Temp, body core temperature; $\mathrm{Hb}$, haemoglobin concentration; $\mathrm{paO}_{2}$, arterial $\mathrm{O}_{2}$ partial pressure; $\mathrm{pvO}_{2}$, mixed venous $\mathrm{O}_{2}$ partial pressure; paCO $\mathrm{P}_{2}$ arterial $\mathrm{CO}_{2}$ partial pressure; $\mathrm{VO}_{2} \mathrm{I}$ and $\mathrm{DO}_{2} \mathrm{l}$, indices of $\mathrm{O}_{2}$ consumption and delivery; $\mathrm{O}_{2}-\mathrm{ER}, \mathrm{O}_{2}$ extraction ratio; $\mathrm{pH}$, pH value; $\mathrm{BE}$, base excess; lactate, lactate concentration; $\mathrm{n}$. a., not applicable. ${ }^{*} P<0.05$ vs. gelatin, $\# P<0.05$ vs. HS, $\S P<0.05$ vs. RS, $+P<0.05$ vs. baseline. 


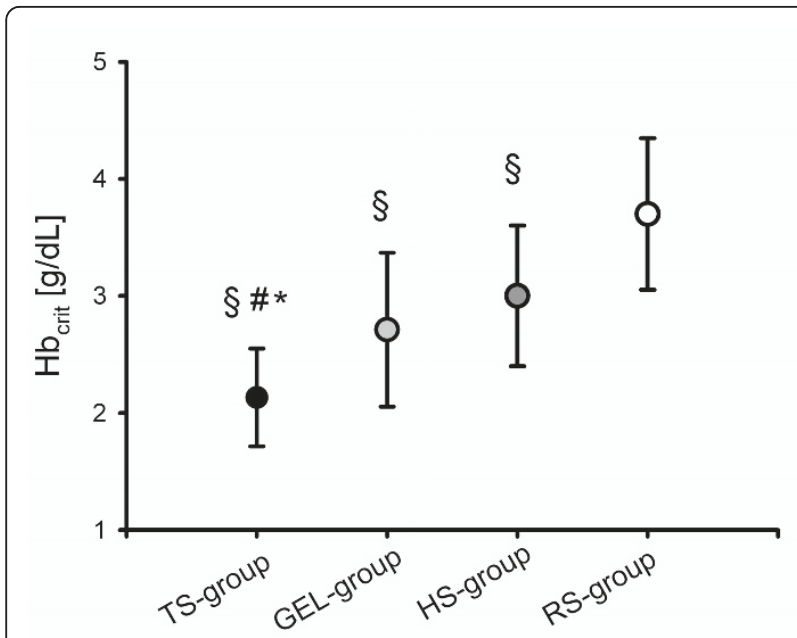

Figure 2 Critical hemoglobin concentration (crit obtained after induction of acute anemia. Anemia was induced using tetrastarch (TS-group, $\mathrm{Hb}_{\text {crit }} 2.1 \pm 0.4 \mathrm{~g} / \mathrm{dL}$ ), gelatin (GEL-group, $\mathrm{Hb}_{\text {crit }} 2.7 \pm 0.6$ $\mathrm{g} / \mathrm{dL}$ ), hetastarch (HS-group, $\mathrm{Hb}_{\text {crit }} 3.0 \pm 0.6 \mathrm{~g} / \mathrm{dL}$ ) or Ringer's solution (RS-group, $\mathrm{Hb}_{\text {crit }} 3.7 \pm 0.6 \mathrm{~g} / \mathrm{dL}$ ). ${ }^{*} P<0.05$ vs. GEL, $\# P<$ 0.05 vs. HS, $\S P<0.05$ vs. RS.

the onset of the critical $\mathrm{DO}_{2}$, lactate concentration was not yet elevated at this time point (see Table 3). The EVLW index (EVLWI) was significantly increased in the RS-group $(P<0.05$ vs. baseline and $P<0.05$ vs. GEL-, HS- and TS-groups, see Figure 3). Consistently, arterial oxygen partial pressure $\left(\mathrm{paO}_{2}\right)$ was decreased in the RS-group $(P<0.05$ vs. baseline and $P<0.05$ vs. GEL-, HS- and TS-groups).

\section{Hemodilution to $\mathrm{Hb} 3.0 \mathrm{~g} / \mathrm{dL}$ ( $H \mathrm{~b}_{\text {crit }}$ of the HS-group)}

As the limit of anemia tolerance was already met at $\mathrm{Hb}$ $3.7 \mathrm{~g} / \mathrm{dL}$ in the RS-group, hemodilution to $3.0 \mathrm{~g} / \mathrm{dL}$ could only be performed in the HS-, GEL- and TS-groups.

A slight increase in CI, HR and SVI and a decrease in MAP and SVRI was observed in these groups, and SVV was significantly reduced compared with baseline. The ITBVI did not change further, but remained elevated above the baseline level $(P<0.05)$. There were no differences between the HS-, GEL and TS-groups in hemodynamic parameters at $\mathrm{Hb} 3.0 \mathrm{~g} / \mathrm{dL}$.

Consistent with progressive hemodilution, $\mathrm{DO}_{2} \mathrm{I}$ further decreased in the remaining HS-, GEL- and TSgroups. In animals hemodiluted with hetastarch, $\mathrm{O}_{2}$ extraction was significantly increased as reflected by lower partial venous oxygen pressure $\left(\mathrm{pvO}_{2}\right)(P<0.05$ vs. baseline and vs. GEL-group) and by a higher $\mathrm{O}_{2}$ extraction ratio $(P<0.05$ vs. baseline and vs. GEL- and TS-groups).

\section{Hemodilution to $\mathrm{Hb} 2.7 \mathrm{~g} / \mathrm{dL}$ (that is, $\mathrm{Hb}_{\text {crit }}$ of the GEL- group)}

Hemodilution could be continued to $\mathrm{Hb} 2.7 \mathrm{~g} / \mathrm{dL}$ in the GEL- and TS-groups. CI did not increase further, but

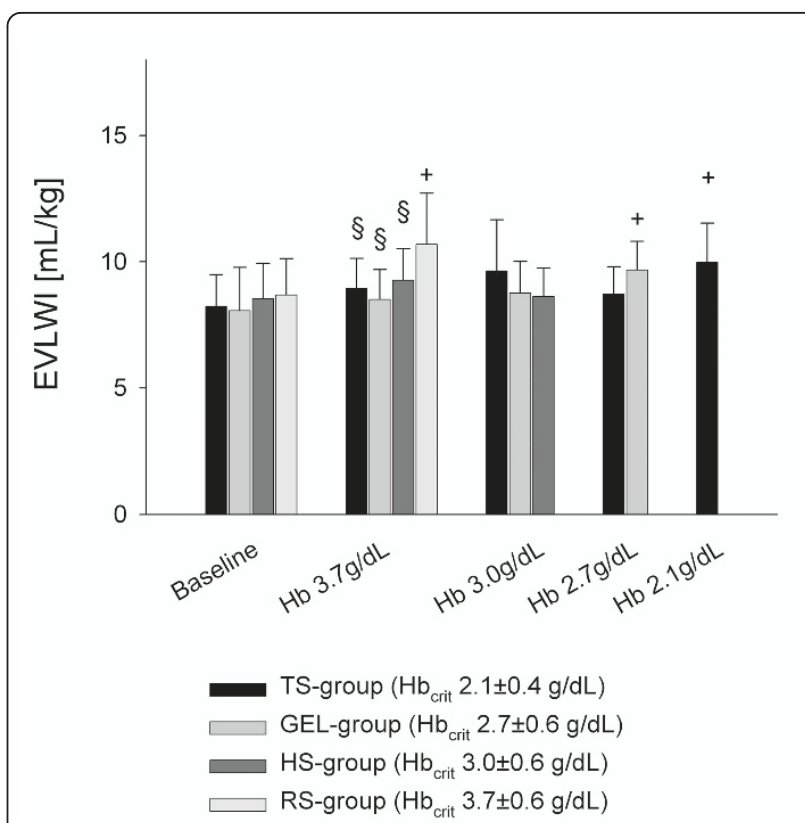

Figure 3 Extravascular lung water index (EVLWI) obtained at baseline after hemodilution to $\mathbf{H b} 3.7 \mathrm{~g} / \mathrm{dL}\left(\mathrm{Hb}_{\text {crit }}\right.$ of the RSgroup), $\mathrm{Hb} 3.0 \mathrm{~g} / \mathrm{dL}$ ( $\mathrm{Hb}_{\text {crit }}$ of the HS-group), $\mathrm{Hb} 2.7$ ( $\mathrm{Hb}_{\text {crit }}$ of the GEL-group) and $\mathrm{Hb} 2.1$ ( $\mathrm{Hb}_{\text {crit }}$ of the TS-group). At Hb $3.7 \mathrm{~g} / \mathrm{dL}$, EVLWI was significantly higher in the RS-group than in the TS-, GEL-, and HS-groups. ${ }^{*} P<0.05$ vs. GEL, $\# P<0.05$ vs. HS, $\S P<0.05$ vs. RS, $+P<0.05$ vs. baseline. $\mathrm{Hb}_{\text {crit, }}$ critical haemoglobin concentration, error bars represent standard deviation of mean.

was now maintained by an elevation of HR in both groups $(P<0.05$ vs. baseline). The MAP and SVRI decreased slightly (non-significant vs. baseline). In both groups, the ITBVI was still higher than at baseline $(P<$ 0.05). However, at $\mathrm{Hb} 2.7 \mathrm{~g} / \mathrm{dL}$, the ITBVI was higher and SVV was lower in the TS-group $(P<0.05$ vs. GELgroup). In the GEL-group, the $\mathrm{O}_{2}$ extraction ratio $\left(\mathrm{O}_{2}\right.$ ER) and EVLWI were elevated when compared with baseline $(P<0.05$, see Figure 3$)$. No further differences between the TS- and GEL-groups were observed in hemodynamic or $\mathrm{O}_{2}$-derived parameters at $\mathrm{Hb} 2.7 \mathrm{~g} / \mathrm{dL}$. Hemodilution to $\mathrm{Hb} 2.1 \mathrm{~g} / \mathrm{dL}$ ( $H \mathrm{~b}_{\text {crit }}$ of the TS-group)

Hemodilution could be performed most extensively with tetrastarch. When animals of the TS-group met their individual Hbcrit at $2.1 \mathrm{~g} / \mathrm{dL}$, the following changes were significantly different to baseline: HR, CI, SVI and EVLWI increased by $30 \%, 90 \%, 36 \%$ and $38 \%$, while SVV, SVRI, $\mathrm{DO}_{2} \mathrm{I}, \mathrm{pvO}_{2}$ and $\mathrm{BE}$ decreased by $52 \%, 64 \%$, $40 \%, 15 \%$ and $72 \%$, respectively $(P<0.05)$. Moreover, $\mathrm{O}_{2}$-ER increased by $38 \%$ and by $40 \%$ (see Table 3 ).

\section{Discussion}

The main findings of the present study are that during acute normovolemic anemia, 1) the choice of the IV fluid has an impact on the maintenance of tissue 
oxygenation as reflected by variation in the extent of anemia tolerance; 2) anemia tolerance is higher when using colloids than when using crystalloids, and 3) among the colloids, tetrastarch (6\% HES 130/0.4) provided higher anemia tolerance than did gelatin $(3.5 \%$ urea-crosslinked polygeline) and hetastarch (6\% HES $450 / 0.7)$. This was reflected by significantly lower values of $\mathrm{Hb}_{\text {crit }}$ and a higher allowable volume of blood exchanged for tetrastarch.

$\mathrm{Hb}_{\text {crit }}$ is the hemoglobin concentration associated with a critical limitation of $\mathrm{O}_{2}$ supply and hallmarks the ultimate tolerance limit of acute normovolemic anemia $[10,11]$. In our previous experimental studies, hemodilution to $\mathrm{Hb}_{\text {crit }}$ was associated with $100 \%$ mortality, if no further treatment (such as elevation of fraction of inspired $\mathrm{O}_{2}$, transfusion of red blood cells, or infusion of artificial $\mathrm{O}_{2}$ carriers) was initiated after institution of critical normovolemic anemia [12-14]. Several authors found $\mathrm{Hb}_{\text {crit }}$ at values between 1.6 and $3.0 \mathrm{~g} / \mathrm{dL}$ [10,12,14-21]. In detail, $\mathrm{Hb}_{\text {crit }}$ was reduced by 1 ) hypothermia (moderate reduction of body core temperature reduces total body $\mathrm{O}_{2}$ demand [15]); 2) hyperoxemia (bioavailability of physically dissolved $\mathrm{O}_{2}$ is excellent in profound anemia $[12,16,17]) ; 3$ ) infusion of norepinephrine (stabilization of coronary perfusion pressure during hemodilution [19]); 4) artificial $\mathrm{O}_{2}$ carriers (maintenance of arterial oxygen content despite reduced hematocrit $[18,22])$, and 5) con-tinuous neuromuscular blockade (lowering skeletal muscular $\mathrm{O}_{2}$ demand [23]).

While different infusion fluids were used for hemodilution in the aforementioned studies, their particular impact on the limit of anemia tolerance has not been fully elucidated. In a similar hemodilution study performed in anesthetized and splenectomised dogs, van der Linden and coworkers found no differences between pentastarch (6\% HES 200/0.5) and a 3\% gelatin preparation in relation to the value of $\mathrm{Hb}_{\text {crit }}$ [20]. However, the typical increase of $\mathrm{CI}$ in compensation for dilutional anemia was absent in that study, which was explained by cardio-depressant effects of the anesthesia regimen employed [20,21].

In the present study, gelatin provided more extensive anemia tolerance than did hetastarch. While the occasional use of hetastarch is predominantly reported by US physicians [24], European physicians rather avoid this fluid due to its adverse effects on coagulation and renal function [25]. Nevertheless, a recent clinical study found a reduction of mortality in trauma victims resuscitated with hetastarch in addition to the advanced trauma life support (ATLS)-standard of care treatment (that is, crystalloids along with blood products) [26].

Hetastarch is a hyperoncotic infusion fluid with a high viscosity in vitro (see Table 1). However, the effects of these properties in vivo are still not fully understood.
While a decrease of plasma viscosity entails the increase of venous return, thereby enabling the hemodynamic compensation of acute anemia [27], recent experimental data suggest that increased plasma viscosity prevents microvascular collapse (for example, after fluid resuscitation from hemorrhagic shock) [6]. Furthermore, the effect of hetastarch on plasma viscosity is limited by its oncotic properties, as the reabsorption of interstitial fluid involves the dilution of viscogenic materials thereby lowering plasma viscosity in the long term [28].

In the present study, animals hemodiluted with hetastarch featured a significantly higher $\mathrm{O}_{2}$-ER at $\mathrm{Hb}_{\text {crit }}$ when compared with animals of the TS- or GEL-group, indicating that macro- and microhemodynamic compensation of anemia was completely exhausted. In other words, hetastarch failed to prevent microcirculatory collapse at an earlier stage of hemodilution than did tetrastarch or gelatin. Although plasma viscosity was not assessed in the present study, it may be assumed that a dilution-related fading of viscogenic potential might have contributed to this result.

Whether fluid resuscitation should be performed with crystalloids or colloids, has been a matter of controversy for decades, and the discussion is still open [29]. Although most crystalloid infusion fluids are plasma-isotonic, they cross the capillary membranes within 20 to 30 minutes of infusion, and most of the volume infused is shifted into the interstitium. To maintain normovolemia with crystalloids, it has been recom-mended to replace an acute blood loss in a ratio of at least 1:3 [30]. However, the exclusive use of crystalloids for volume replacement results in edema formation and may thereby compromise tissue oxygenation [31]. Actually, excessive tissue hydration due to pure crystalloid volume replacement in the perioperative phase was held responsible for many postoperative complications including increased incidence of anastomotic dehiscence in abdominal surgery, postoperative vomiting and orthostatic dysregulation [32,33]. Moreover, an experimental study in pigs subjected to colon anastomosis surgery found that microcirculatory blood flow and oxygen tension in perianastomotic colon tissue were increased in animals infused with tetrastarch when compared with crystalloid fluid management [34]. This finding was explained by homogenisation of mucosal microcirculatory blood flow after infusion of tetrastarch.

In the present study, with Ringer's solution, the exchange of blood was associated with increased pulmonary edema formation (elevated EVLWI) and an impairment of pulmonary gas exchange (decreased $\mathrm{paO}_{2}$ ). This phenomenon was not observed in animals hemodiluted with gelatin, hetastarch or tetrastarch. Although EVLWI merely reflects the degree of tissue hydration at the site of pulmonary circulation, it may be 
supposed that a relevant edema formation also occurred in peripheral $\mathrm{O}_{2}$-consuming tissues: while $\mathrm{O}_{2}$ extraction increased consistently in animals hemodiluted with any of these colloids, $\mathrm{O}_{2}$ extraction was not increased when animals of the RS-group met their individual $\mathrm{Hb}_{\text {crit }}$. As this finding may reflect a microcirculatory disorder related to excessive tissue hydration, we conclude that the comparatively early $\mathrm{VO}_{2}$-decrease in the $\mathrm{RS}$-group might, in addition to the anemia-related restriction of the $\mathrm{O}_{2}$ transport capacity, also be attributable to an edema-related impairment of $\mathrm{O}_{2}$ uptake at the site of pulmonary and peripheral microcirculation.

A certain transcapillary filtration rate is also characteristic for gelatin preparations [35]. Their low molecular weight (30 to $40 \mathrm{kDa}$ ) entails a rapid passage into the interstitial space and a rapid clearance by glomerular filtration, finally reducing volume efficacy to $80 \%$ (that is, $20 \%$ extravasation rate). Consistently, EVLWI increased much later in the GEL-group than in the RS-group. Moreover, when hemodilution was continued below $\mathrm{Hb}$ $3.7 \mathrm{~g} / \mathrm{dL}, \mathrm{O}_{2}$ extraction could be augmented, reflecting that microcirculatory function might have been maintained more adequately than in the RS-group.

The strict maintenance of normovolemia is essential for adequate hemodynamic compensation of acute anemia, that is, for the increase in cardiac output. However, one weakness of the present protocol is the verification of normovolemia at time points characterized by extreme anemia. During extreme hemodilution, the kinetic of ICG elimination is significantly altered by dilution of albumin and increased cardiac output. Therefore, a measurement of circulating blood volume at this point would yield results not comparable with the baseline measurement [7], so normovolemia was deduced from clinically assessable parameters, for example, ITBVI and SVV.

While ITBVI represents LV preload, decreases in SVV reflect adequate volume responsiveness. After hemodilution with either fluid, ITBVI increased above the baseline level, indicating that volume replacement was adequate to achieve macro-hemodynamic compensation of acute anemia (increase of venous return to the heart, augmentation of LV preload). However, the increase of ITBVI was more pronounced in the TS- and HS-groups. In the course of hemodilution, SVV decreased in the TS-, GEL-, and HSgroups. In the RS-group, SVV remained unchanged and at $\mathrm{Hb}_{\text {crit }}$, it was significantly lower than in the HS-group. Both findings reflect that volume responsiveness was most strongly expressed in the starch groups. In the RS-group, however, volume responsiveness and volume efficacy were limited by partial extravasation despite infusion of $3 \mathrm{~mL}$ RS per $\mathrm{mL}$ blood withdrawn.

Although this procedure provided adequate LV preload for hemodynamic compensation of acute anemia, it may be argued that the infusion of higher volumes of
RS (4 or $5 \mathrm{~mL}$ per $\mathrm{mL}$ blood withdrawn) might have elevated intravascular volume and might have improved volume responsiveness. On the other hand, extravasation and tissue hydration would have exacerbated and might have further impaired $\mathrm{O}_{2}$ uptake and tissue oxygenation.

Likewise, the volume effect of gelatin appeared to be limited by its short intravascular half-life and its extravasation tendency. No differences between the colloid groups were observed in LV preload or volume responsiveness until hemodilution to $\mathrm{Hb} 3.0 \mathrm{~g} / \mathrm{dL}$. However, after continuation of hemodilution to $2.7 \mathrm{~g} / \mathrm{dL}$, ITBVI tended to decrease and SVV to increase in the GELgroup, and both parameters differed significantly from the TS-group. Nevertheless, animals of the GEL-group still featured sufficient hemodynamic compensation of acute anemia (CI, SVI and ITBVI remained increased vs. baseline), which precludes that they were actually hypovolemic.

These findings indicate that 1) the infusion of $1.2 \mathrm{~mL}$ gelatin per $\mathrm{mL}$ blood withdrawn was adequate to maintain LV preload and volume responsiveness over a wide range of the hemodilution protocol; 2) in spite of this, the volume effect of gelatin could not be sustained until the end of the protocol, and 3) that extravasation and fading volume responsiveness might have compromised microvascular perfusion, which finally caused animals of the GEL-group to earlier achieve Hbcrit than the TSgroup.

We chose $\mathrm{Hb}_{\text {crit }}$ as the primary endpoint of the hemodilution protocol. In our previous studies, lactate concentrations began to increase 60 to 90 minutes after institution of $\mathrm{Hb}_{\text {crit }}[12-14,19]$. Of note, we employed the identical hemodilution protocol in the present study but animals were killed immediately after achievement of $\mathrm{Hb}_{\text {crit }}$, so that elevated lactate concentrations could not yet be expected.

In the present experimental model, the effects of the investigated infusion fluids became apparent when the $\mathrm{O}_{2}$ transport capacity was driven to its critical limit. Whether differences between the groups existed already at less severe degrees of anemia, is difficult to conclude.

However, a clinical investigation in healthy volunteers undergoing moderate hemodilution with different HES preparations (HES 130/0.4, HES 70/0.5 or HES 200/0.5) demonstrated that tetrastarch provided the most sustained increase of tissue $\mathrm{O}_{2}$ partial pressure $\left(\mathrm{tpO}_{2}\right)$ [36].

In summary, our data suggest that the choice of the IV infusion fluid used for acellular volume replacement has an impact on the maintenance of $\mathrm{O}_{2}$ supply. Among the fluids investigated in the present study, tetrastarch (HES 130/0.4) provided the most extensive anemia tolerance when compared with gelatin, hetastarch or Ringer's solution. While the exact underlying mechanism 
remains to be elucidated, extravasation and formation of interstitial edema were associated with decreased anemia tolerance, indicating that the microcirculatory effects of the fluids investigated had a major impact on tissue oxygenation.

\section{Conclusions}

During acellular treatment of an acute blood loss in lieu of allogeneic blood transfusions (for example, correction of hypovolemia, bridging an unexpected massive blood loss, or declining of transfusion for religious reasons), modern low-molecular tetrastarch preparations (such as HES 130/ 0.4 ) appear most suitable to maintain $\mathrm{O}_{2}$ supply over a wide range of levels of acute normovolemic anemia.

\section{Key messages}

- The correction of hypovolemia with acellular fluids implies the dilution of the cell mass remaining in the vasculature (acute normovolemic anemia).

- Sustainment of normovolemia and nutritive microcirculatory blood flow has a major impact on the maintenance of tissue oxygenation and thereby on the tolerance of acute anemia.

- The fluids investigated in the present study (tetrastarch, gelatin, hetastarch and Ringer's Solution) differed in their volume effects and their extravasation rate and might thereby have influenced microcirculatory function.

- In profound anemia, modern low-molecular tetrastarch preparations were most advantageous to maintain normovolemia as well as tissue oxygenation.

\begin{abstract}
Abbreviations
ANOVA: analysis of variance; BE: base excess; BSA: body surface area; BVI: circulating blood volume (indexed to $\mathrm{BSA}$ ); $\mathrm{CaO}_{2}$ : arterial oxygen content; $\mathrm{Cl}$ : cardiac index (cardiac output indexed to $\mathrm{BSA}$ ); $\mathrm{DO}_{2}$ : oxygen delivery; EVLWI: extravascular lung water (indexed to BSA); $\mathrm{Hb}_{\text {crit: }}$ : critical hemoglobin concentration; HES: hydroxyethyl starch; HR: heart rate; IV: intravenous; ICG: indocyaningreen; ITBVI: intrathoracic blood volume (indexed to BSA); MAP: mean arterial pressure; MPAP: mean pulmonary arterial pressure; $\mathrm{O}_{2}$-ER: oxygen extraction ratio; $\mathrm{paO}_{2}$ : arterial oxygen partial pressure; PCWP: pulmonary capillary wedge pressure; $\mathrm{PVO}_{2}$ : mixed-venous oxygen partial pressure; SVI: stroke volume (indexed to BSA); SVRI: systemic vascular resistance (indexed to $\mathrm{BSA}$ ); $\mathrm{SW}$ : stroke volume variation; $\mathrm{VO}_{2}$ : total body oxygen consumption.
\end{abstract}

\section{Acknowledgements}

The authors would like to thank Mr W Daut and his team for their excellent performance in animal care and $\mathrm{Mr} \mathrm{H}$ Winkelmeier for his valuable technical assistance.

\section{Author details}

${ }^{1}$ Clinic of Anesthesiology, Intensive Care Medicine and Pain Management, J. W. Goethe-University Hospital Frankfurt, Theodor-Stern-Kai 7, Frankfurt/Main, 60590, Germany. ${ }^{2}$ Clinic of Anesthesiology, Ludwig Maximilians University Hospital, Marchioninistraße 15, Munich, 81377, Germany. ${ }^{3} \mathrm{Clinic}$ of Anesthesiology, Surgical Intensive Care Medicine and Pain Management, Krankenhaus Nordwest, Steinbacher Hohl 2-26, Frankfurt/Main, 60488 Germany.

\section{Authors' contributions}

$\mathrm{AP}, \mathrm{SK}, \mathrm{HK}, \mathrm{OH}$ and $\mathrm{ML}$ carried out the experiments. SK coordinated data acquisition during the experiments and built up the database. PS performed the statistical analysis. AP, BZ and $\mathrm{OH}$ conceived the study and participated in its design. $\mathrm{BZ}$ and $\mathrm{OH}$ helped to draft the manuscript. All authors read and approved the final manuscript.

\section{Competing interests}

The study was sponsored by a research grant from the Else KrönerFresenius-Foundation, Bad Homburg, Germany. The authors declare that they have no further competing interests.

Received: 23 December 2011 Revised: 2 March 2012

Accepted: 30 April 2012 Published: 30 April 2012

\section{References}

1. Messmer KF: Acceptable hematocrit levels in surgical patients. World J Surg 1987, 11:41-46.

2. Madjdpour C, Spahn DR, Weiskopf RB: Anemia and perioperative red blood cell transfusion: a matter of tolerance. Crit Care Med 2006, 34:S102-S108.

3. Pape A, Habler O: Alternatives to allogeneic blood transfusions. Best Pract Res Clin Anaesthesiol 2007, 21:221-239.

4. Habler OP, Messmer KF: The physiology of oxygen transport. Transfus Sci 1997, 18:425-435.

5. Schumacker PT, Cain SM: The concept of a critical oxygen delivery. Intensive Care Med 1987, 13:223-229.

6. Cabrales P, Tsai AG, Intaglietta M: Increased plasma viscosity prolongs microhemodynamic conditions during small volume resuscitation from hemorrhagic shock. Resuscitation 2008, 77:379-386.

7. Haller M, Akbulut C, Brechtelsbauer H, Fett W, Briegel J, Finsterer U, Peter K: Determination of plasma volume with indocyanine green in man. Life Sci 1993, 53:1597-1604.

8. Holt JP, Rhode EA, Kines $\mathrm{H}$ : Ventricular volumes and body weight in mammals. Am J Physiol 1968, 215:704-715.

9. Meier JM, Woelkhammer S, Habler OP: The Deltacrit-System (DCS) - a new method for online-determination of critical $\mathrm{VO}_{2}$-decline. Comput Biol Med 2003, 33:395-405.

10. Cain SM: Oxygen delivery and uptake in dogs during anemic and hypoxic hypoxia. J Appl Physiol 1977, 42:228-234.

11. De Backer D: VO2/DO2 relationship: how to get rid of methodological pitfalls? Intensive Care Med 2000, 26:1719-1722.

12. Meier JM, Kemming Gl, Kisch-Wedel H, Wolkhammer S, Habler OP: Hyperoxic ventilation reduces 6-hour mortality at the critical hemoglobin concentration. Anesthesiology 2004, 100:70-76.

13. Meier JM, Pape A, Lauscher P, Zwissler B, Habler OP: Hyperoxia in lethal methemoglobinemia - effects on $\mathrm{O}_{2}$ transport, tissue oxygenation and survival in pigs. Crit Care Med 2005, 33:1582-1588.

14. Pape A, Kertscho $H$, Meier J, Horn O, Laout M, Steche M, Lossen M, Theisen A, Zwissler B, Habler O: Improved short-term survival with polyethylene glycol modified hemoglobin liposomes in critical normovolemic anemia. Intensive Care Med 2008, 34:1534-1543.

15. Perez-de-Sa V, Roscher R, Cunha-Goncalves D, Larsson A, Werner O: Mild hypothermia has minimal effects on the tolerance to severe progressive normovolemic anemia in Swine. Anesthesiology 2002, 97:1189-1197.

16. Pape A, Meier J, Kertscho H, Steche M, Laout M, Schwerdel F, Wedel M, Zwissler B, Habler OP: Hyperoxic ventilation increases the tolerance of acute normovolemic anemia in anesthetized pigs. Crit Care Med 2006, 34:1475-1482

17. Kemming Gl, Meisner FG, Kleen MS, Meier JM, Tillmanns JH, Hutter JW, Wojtczyk CJ, Packert KB, Bottino DA, Habler OP: Hyperoxic ventilation at the critical haematocrit. Resuscitation 2003, 56:289-297.

18. Meisner FG, Kemming Gl, Habler OP, Kleen MS, Tillmanns JH, Hutter JW Bottino DA, Thein E, Meier JM, Wojtczyk CJ, Pape A, Messmer K: Diaspirin crosslinked hemoglobin enables extreme hemodilution beyond the critical hematocrit. Crit Care Med 2001, 29:829-838.

19. Meier J, Pape A, Loniewska D, Lauscher P, Kertscho H, Zwissler B, Habler O: Norepinephrine increases tolerance to acute anemia. Crit Care Med 2007, 35:1484-1492.

20. van der Linden P, Schmartz D, De Groote F, Mathieu N, Willaert P, Rausin I, Vincent JL: Critical haemoglobin concentration in anaesthetized dogs: comparison of two plasma substitutes. Br J Anaesth 1998, 81:556-562. 
21. van der Linden $P$, De Hert S, Mathieu N, Degroote F, Schmartz D, Zhang H, Vincent JL: Tolerance to acute isovolemic hemodilution. Effect of anesthetic depth. Anesthesiology 2003, 99:97-104.

22. Habler OP, Kleen MS, Hutter JW, Podtschaske AH, Tiede M, Kemming GI, Welte MV, Corso CO, Batra S, Keipert PE, Faithfull NS, Messmer KF: Hemodilution and intravenous perflubron emulsion as an alternative to blood transfusion: effects on tissue oxygenation during profound hemodilution in anesthetized dogs. Transfusion 1998, 38:145-155.

23. Pape A, Kertscho H, Stein P, Lossen M, Horn O, Kutschker S, Zwissler B, Habler O: Neuromuscular blockade with rocuronium bromide increases the tolerance of acute normovolemic anemia in anesthetized pigs. Eur Surg Res 2011, 48:16-25.

24. Treib J, Baron JF, Grauer MT, Strauss RG: An international view of hydroxyethyl starches. Intensive Care Med 1999, 25:258-268.

25. Ertmer C, Rehberg S, Van AH, Westphal M: Relevance of non-albumin colloids in intensive care medicine. Best Pract Res Clin Anaesthesiol 2009, 23:193-212.

26. Ogilvie MP, Pereira BM, McKenney MG, McMahon PJ, Manning RJ, Namias N, Livingstone AS, Schulman Cl, Proctor KG: First report on safety and efficacy of hetastarch solution for initial fluid resuscitation at a level 1 trauma center. J Am Coll Surg 2010, 210:870-872.

27. Messmer K, Sunder-Plassmann L: Hemodilution. Prog Surg 1974, 13:208-245.

28. Villela N, Salazar Vasquez B, Intaglietta M: Microcirculatory effects of intravenous fluids in critical illness: plasma expansion beyond cystalloids and colloids. Curr Opin Anaesthesiol 2009, 22:163-167.

29. Ertmer C, Kampmeier T, Rehberg S, Lange M: Fluid resuscitation in multiple trauma patients. Curr Opin Anaesthesiol 2011, 24:202-208.

30. Spahn DR, Cerny V, Coats TJ, Duranteau J, Fernández-Mondéjar E, Gordini G, Stahel PF, Hunt BJ, Komadina R, Neugebauer E, Ozier Y, Riddez L, Schultz A, Vincent $J$, Rossaint R, Task Force for Advanced Bleeding Care in Trauma: Management of bleeding following major trauma: a European guideline. Crit Care 2007, 11:412

31. Tollofsrud S, Svennevig JL, Breivik H, Kongsgaard U, Ozer M, Hysing E, Mohr B, Seem E, Geiran O, Abdelnour M: Fluid balance and pulmonary functions during and after coronary artery bypass surgery: Ringer's acetate compared with dextran, polygeline, or albumin. Acta Anaesthesiol Scand 1995, 39:671-677.

32. Moretti EW, Robertson KM, El Moalem H, Gan TJ: Intraoperative colloid administration reduces postoperative nausea and vomiting and improves postoperative outcomes compared with crystalloid administration. Anesth Analg 2003, 96:611-7.

33. Grocott MP, Mythen MG, Gan TJ: Perioperative fluid management and clinical outcomes in adults. Anesth Analg 2005, 100:1093-1106.

34. Kimberger O, Arnberger M, Brandt S, Plock J, Sigurdsson GH, Kurz A, Hiltebrand L: Goal-directed colloid administration improves the microcirculation of healthy and perianastomotic colon. Anesthesiology 2009, 110:496-504

35. Holbeck S, Grande PO: Effects on capillary fluid permeability and fluid exchange of albumin, dextran, gelatin, and hydroxyethyl starch in cat skeletal muscle. Crit Care Med 2000, 28:1089-1095.

36. Stand $T$, Burmeister MA, Schroeder F, Currlin E, Schulte am EJ, Freitag M, Schulte am EJ: Hydroxyethyl starch (HES) 130/0.4 provides larger and faster increases in tissue oxygen tension in comparison with prehemodilution values than HES $70 / 0.5$ or HES $200 / 0.5$ in volunteers undergoing acute normovolemic hemodilution. Anesth Analg 2003, 96:936-943.

doi:10.1186/cc11324

Cite this article as: Pape et al:: The choice of the intravenous fluid influences the tolerance of acute normovolemic anemia in anesthetized domestic pigs. Critical Care 2012 16:R69.

\section{Submit your next manuscript to BioMed Central and take full advantage of:}

- Convenient online submission

- Thorough peer review

- No space constraints or color figure charges

- Immediate publication on acceptance

- Inclusion in PubMed, CAS, Scopus and Google Scholar

- Research which is freely available for redistribution

Submit your manuscript at www.biomedcentral.com/submit
Biomed Central 